\title{
Inteligência Social de uma cidade: escutar e aprender os contextos narrados pelo cidadão em meios as redes sociais
}

\author{
Viviani Rios Kwecko, IFRS, viviani.kwecko@riogrande.ifrs.edu.br \\ Fernando Pereira de Tolêdo, FURG, ftoledo@furg.br \\ Gabriel Moraes, FURG, g.moraes@furg.br \\ Silvia Silva da Costa Botelho, FURG, silviacb@furg.br
}

Resumo: Este estudo apresenta como objetivo a criação de um modelo tecnológico de escuta e interação de modo a qualificar a relação da população com sua cidade via produção de conhecimento em redes sociais digitais. Essa modelagem é composta por três etapas: um sistema de mineração de dados no qual combinamos diversas técnicas para a descoberta de conhecimento, resultando na escuta coletiva do cidadão, gerando a partir disso, termos representativos "sociais" (de todos); a compreensão destes termos como representações sociais de contextos, através de uma Análise limplicativa Hierárquica (A.S.I.) buscando a visualização a similaridade de diferentes percursos associativos; e uma última etapa de intervencão social, durante a qual é prevista a atuação de um chatbot visando uma conscientização da papulação acerca inteligência social da cidade.

Palavras-chave: Modelo Tecnológico, Redes Sociais Digitais, Descoberta de Conhecimento Análise limplicativa Hierárquica, Chatbot

\section{Social Intelligence of a city: to listen and to learn the contexts narrated by the citizen in means the social networks}

\begin{abstract}
This study aims to create a technological model of listening and interaction in order to qualify the relationship of the population with their city through the production of knowledge in digital social networks. This modeling is composed of three stages: a data mining system in which we combine several techniques for the discovery of knowledge, resulting in the collective listening of the citizen, generating from this, representative "social" (all) terms; the understanding of these terms as social representations of contexts, through a hierarchical hierarchical analysis (A.S.I.) seeking to visualize the similarity of different associative paths; and a last stage of social intervention, during which it is expected to perform a chatbot aimed at raising awareness about the social intelligence of the city.
\end{abstract}

Keywords: Technological Model, Digital Social Networks, Knowledge Discovery Hierarchical limplicative Analysis, Chatbot

\section{Introdução}

Atualmente as implicações do desenvolvimento das Tecnologias Digitais de Informação e de Comunicação (TDIC) nos colocam diante do desafio de pensar como a interconexão mundial dos computadores afeta a cultura da cidade e num movimento inverso, como a cidade modifica a utilização dessas tecnologias. Ao deslocamos nosso 


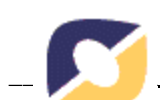

olhar para problematizar algumas dessas relações, percebemos a natureza interdisciplinar de nosso objeto de pesquisas. Assim reunimos as áreas da Computação Social, da Educação, da Sociologia e da Arte para compor uma pesquisa que transita entre o social, no desenvolvimento de conhecimento; a aplicação, por implementar os conceitos na prática; e o desenvolvimento de uma técnica (modelo de mediação tecnológica)

O objetivo deste artigo é explicitar como se desenvolveu a modelagem do sistema tecnológico de escuta e intervenção de modo a qualificar a relação da população com sua cidade via produção de conhecimento social em redes sociais digitais. A situação problema que emerge é como integrar a opinião da população nas redes sociais à inteligência da cidade, sem que essa interação reproduza um repositório de problemas, mas sim represente processos de solução sociais. Uma possível solução apontada é fomentar discussões acerca dos problemas da cidade nas redes sociais.

É importante esclarecer uma das grandes questões que mobilizam essa pesquisa, a educação tácita, não regulada, que se produz cada dia sem que tomemos consciência dela, mas que é fundamental na formação dos cidadãos. O campo da educação neste trabalho, difere de pesquisas que abordam os processos institucionais (sistema pedagógico) ou o ato de educar (ensino e aprendizagem), pois tomamos o conceito em seu sentido amplo. A finalidade deste estudo determina o objeto de investigação que, por sua vez, relaciona-se diretamente com os campos discursivos que constroem cotidianamente os núcleos de saberes sociais de uma cidade, manifestos em contextos de interações digitais. Designamos tais saberes sociais não como essência fundadora e definitiva da educação, mas como um conjunto de relações que permite a constituição do sentimento de pertencimento a uma comunidade. Os focos principais são as aprendizagens construídas em processos sociais coletivos/participativos onde o saber não é gerado em estruturas formais de ensino, mas no campo da educação informal ${ }^{1}$ (Libâneo, 2010). Falar sobre as relações entre aprendizagem e saber fora do sistema regular de ensino implica em participar do amplo debate epistemológico sobre a produção de conhecimento no mundo contemporâneo.

Esta escolha justifica-se por acreditarmos que, através destes conjuntos de argumentações acerca da experiência vivida a população partilha saberes, crenças e traços de comportamentos. O desafio é entender os pontos de convergência e divergência entre 0 espaço urbano da cidade e o espaço virtual das redes sociais digitais, aparentemente tão distintos. Assim, partindo do pressuposto que as informações em redes digitais revelam inteligências coletivas que organizadas e problematizadas podem transformar as opiniões da população em conhecimentos sociais, a intenção desta pesquisa está em identificar quem é essa cidade inteligente. Deste modo, o presente artigo apresenta a modelagem de um sistema de conexão entre a prática cotidiana dos usuários em postar suas opiniões nas redes sociais digitais e os indicadores de inteligência de uma cidade. Para tanto, organizamos o modelo de produção de inteligência social a partir das seguintes etapas: (i) escutando o cidadão, (ii) contextos narrados e (iii) chatbot (Figura 1).

Este artigo encontra-se estruturado da seguinte forma: na seção dois são descritos conceitos e modelos que embasaram o desenvolvimento da proposta; na seção três são apresentados os métodos adotados para sua execução; na seção quatro são apresentadas as discussões e por fim, na seção 5 são apresentadas as considerações referentes à pesquisa.

1 A educação informal corresponderia a ações e influências exercidas pelo ambiente socio- cultural, e que se desenvolve por meio das relações dos indivíduos e grupos das quais resultam conhecimentos, experiências, práticas, mas que não estão ligadas especificamente a uma instituiç̧ão (Libâneo, 2010, p. 31). 


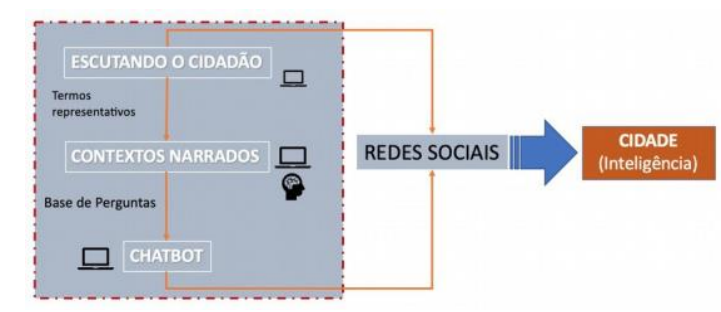

Figura 1 - Modelo de produção de inteligência em redes sociais

\section{Fundamentação Teórica}

O European Smart Cities (Gaspar, 2016), é uma metodologia que descreve a inteligência da cidade através de seis características (economia inteligente, pessoas inteligentes, governança inteligente, mobilidade inteligente, meio ambiente inteligente, vida inteligente), 31 fatores e 74 indicadores. No Brasil, a Urban Systems (2018), apresenta o Ranking Connected Smart Cities que verifica o nível de inteligência de uma cidade a partir de 11 eixos, (mobilidade, urbanismo, meio ambiente, energia, tecnologia e inovação, economia, educação, saúde, segurança, empreendedorismo e governança) e 73 indicadores. Outras iniciativas vêm sendo consideradas, como por exemplo, IESE Cities in Motion (lese Business School; University of Navarra, 2016); o projeto "Brasil 2030: Cidades inteligentes e humanas", coordenado pela Rede Brasileira de Cidades Inteligentes e Humanas em parceria com a Frente Nacional de Prefeitos, que considera a verificação de 4 eixos (Governança e Arquitetura, Urbanismo e Antropologia, Tecnologia e Segurança) e 205 indicadores; e os estudos do brasileiro Afonso et al. (2013) que propõe, para além dos indicadores, a constituição de um índice de maturidade para cidades inteligentes.

O Intelligent Community Forum (ICF), define como cidades e regiões inteligentes as que utilizam a tecnologia não para economizar dinheiro ou fazer as coisas funcionarem melhor, mas também para aumentar a participação dos cidadãos e tornar os lugares atraentes para viver e trabalhar. Estudos discutem essa aproximação do cidadão com a inteligência da cidade a partir de processos informatizados sensíveis ao contexto (Gabrys, 2014) e (Granier e Kudo, 2016).

Diferente das outras abordagens, neste estudo é apresentada uma análise da participaçãao da população na produção de inteligência das cidades, observando sobretudo quais enfoques tecnológicos são utilizados para essa vinculação. Para tanto são referidos o uso de análise web, mineração de dados, neural networks, deep learning e machine learning para estabelecer as fontes, os atores, as atividades, as redes e as estratégias de comunicação com a população. Han et al. (2011) observam que a mineração de dados na web estruturam-se nos enfoques de: (i) Mineração de Estrutura (Web Structure Mining), quando o conhecimento decorre da organização e da referência cruzada de ligações, em especial através de hiperlinks; (ii) Mineração de Uso (Web Usage Mining), gerando padrões com o uso dos registros de navegação dos usuários (logs); e (ii) Mineração de Conteúdo (Web Content Mining), no qual o conhecimento decorre do conteúdo dos documentos e de seus metadados.

Deep Learning (DL) representa um conjunto de técnicas de Machine Learning baseadas em redes neurais, capazes de extrair e aprender de forma a "entender" os dados 
(Lecun, 2015). O termo "deep" surgiu no contexto de que as redes neurais utilizadas para realizar esse aprendizado possuem múltiplas camadas, tornando-se redes profundas. Isso acontece, pois cada camada da rede é responsável por extrair determinadas características dos dados de entrada Goodfellow (2016). No contexto de processamento de texto, são capazes de prever Poplin et al. (2018), classificar He et al. (2016), extrair contexto Hossain, et al. (2019), dentre outras aplicaç,ões.

Análise Estatística Implicativa (A.S.I.), um método estatístico multidimensional aplicado à pesquisas qualitativas que busca uma análise hierárquica de similaridade ou semelhanças (Almeida et al., 2000) entre um conjunto de dados. Segundo Couturier et al. (2004) essa abordagem permite ao pesquisador (i) extrair de um conjunto de dados regras de associação entre variáveis; (ii) fornecer um índice de qualidade de associação; e (iii) representar uma estruturação das variáveis obtida por meio destas regras. Aplicamos a A.S.I através do uso do software de Classificação Hierárquica Implicativa e Coesiva CHIC (Classification Hierarchique Implicative et Cohesitive - Versão 6.0, 2012). No âmbito do mapeamento de concepcõoes, a A.S.I vem sendo aplicada na Educação principalmente para análise do letramento estatístico (Giordano, 2019; Fotiadis e Anastasiadou, 2019), para verificação de processos de ensino e aprendizagens (De Almeida Costa et al., 2018) e de formação de professores (Rodrigues, 2018); quanto ao estudo do comportamento social dos indivíduos, esse modelo estatístico tem contribuído na identificação de padrões (Anastasiadou e Papadaki, 2019), (Ono et al., 2014), para avaliação de modelos de recomendação ( Paez Candelaria et al., 2018) e na verificação do impacto do uso ferramentas tecnológicas (Rakotomalala et al., 2019).

Ao integrarmos as concepc,ões obtidas com a A.S.I. a uma ferramenta de interação como um mecanismo de chatbot, estamos permitindo um novo processo de escuta/respostas. Através deste processo seria possível gerar às indagaç,ões e ou conflitar o usuário de modo a promover a consciência social. Exemplos de chatbots podem ser aplicados nas áreas de saúde (Almeida Junior, 2017), negócios (Vendrúscolo e Moré, 2018) e educação (Graf et al., 2017), (Kuyven et al., 2018). O Citybot: : a chatbot interface for a smart city on Behance é uma interface projetada para dispositivos móveis concentrada particularmente em tornar mais inteligente Mumbai, a mais importante cidade da Índia. A ideia-chave é construir uma cidade inteligente em torno das pessoas fazendo com que a cidade fale com a população, principalmente quanto a oferta de informações aos cidadãos. No estudo acerca do Estado da Arte das pesquisas sobre Chatbot desenvolvido por (Sônego et al., 2018), identificamos uma pesquisa que se aproximam do nosso objeto de estudo.

\section{Procedimentos Metodológicos}

Nesta pesquisa propomos um Modelo de Mineração de Dados (de Postagens do Facebook) para Inferência da Inteligência do Território fundamentado nos conceitos tecnológicos definidos nas seções acima e organizado em três etapas: i) a classificação de padrões com base em técnicas de Aprendizado Profundo (Deep Learning - DL) e ii) a análise da representaç̧ão de contextos e visualizaçãa de diferentes percursos associativos nas publicacõões por meio da Análise Estatística Implicativa (A.S.I.) de modo a gerar uma base de conhecimento para intervenções (Top- $\mathrm{N}$ <intenç̃oses de entrada>) para um chatbot.

2 https://www.behance.net/gallery/50882425/CITYBOT-A-chatbot-interface-for-a-smart-city 
$\mathrm{Na}$ etapa de Escuta do Cidadão ocorre o pré-processamento das postagens, anotação dos dados e obtenção dos termos representativos, como um conjunto de técnicas e ferramentas de mineração capazes de inferir o grau de inteligência de um território a partir de postagens da referida rede social. Para a constituição dos Contextos Narrados utilizamos uma adaptação da Análise Estatística Implicativa (A.S.I.), um método estatístico multidimensional aplicado à pesquisas qualitativas que busca uma análise hierárquica de similaridade ou semelhanças (Almeida et al., 2000). O Chatbot, que encontra-se em desenvolvimento, utilizará a base de conhecimento das concepcōoes organizadas a partir para realizar sua intervenção previstas sob duas formas: pela oferta de informacọes acerca da temática discutida ou por uma indagação vincular.

\section{Resultados e discussões}

Os resultados obtidos aqui limitam-se a apresentação da organização das técnicas que compõem o modelo proposta para organização da Inteligência Social da Cidade.

\subsection{Descoberta de Conhecimento em Bancos de Dados (Knowledge Discovery in Databases - KDD) para categorização das postagens e da polaridade de sentimento}

O procedimento de Mineração de Dados é um processo que pode ser aplicado a qualquer problema que requeira a identificação de padrões em dados. Como resultado, é possível obter a transformação de um mapeamento de dados brutos em arranjos de informação estruturada que possibilitam a identificação de novos padrões válidos e potencialmente úteis para a configuração de referenciais preditivos de ações futuras. Para tanto, aplicamos um modelo (Figura 2) de rede neural profunda C-LSTM, utilizado para a classificação dos dados entre 11 eixos, além de permitir a extração da polaridade a nível de sentimento das postagens (conceito de granularidade grossa ${ }^{3}$ ).

Conforme abordagem proposta envolvendo DL, se fez necessário a obtenção de um conjunto de postagens anotadas e classificadas num total de 42.062 mil publicações. $\mathrm{Na}$ fase de treinamento do modelo foi utilizado um dataset com 1138 publicaç,ões anotadas a partir do sistema Web (dataset de classificação). A acurácia atingida no conjunto de teste foi de $76.32 \%$, quanto à classificação nos 11 eixos, para a amostra utilizada durante o treinamento da rede. Este valor é satisfatório, e justificável devido ao número de amostras que foram dadas como entrada da rede (1138 entradas, sendo somente 682 para treino). De posse da catalogaç̧ão das postagens, procedemos com a etapa II de classificaçáa do sentimento em negativo, positivo ou neutro, na qual foi atingida uma acurácia de $80,70 \%$. O resultado levemente superior (somente 4,68\%), considerando que o número de classes a serem preditas diminuiu de 11 para 3 sugere que a identificação da polaridade de sentimento pela rede é uma tarefa mais complexa quando comparada a classificação de eixos de cidades inteligentes.

De posse do conjunto de postagens para cada aspecto do Ranking Connected Smart Cities procede-se a identificação da co-ocorrência das palavras que combinadas resultaram em termos representativos para cada um dos 11 eixos, e em 3 aspectos de sentimento.

3 Classificação de polaridade em positivo, negativo e neutro 


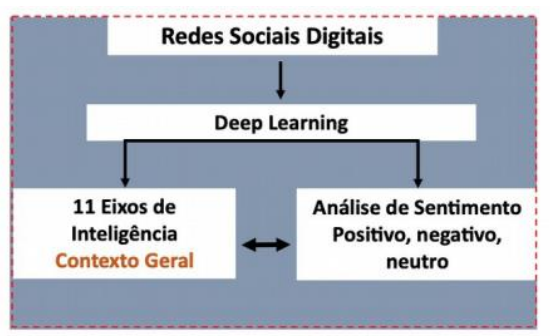

Figura 2 - Etapa de Escuta do Cidadão

A posteriori estes termos são (re)organizados em meta-categorias semânticas para novo processamento durante a etapa de exploração dos contextos específicos por meio da Análise Estatística Implicativa (A.S.I.) que processam Grafo de Similaridade, a Árvore Implicativa e Coesitiva.

\subsection{Análise Estatística Implicativa (A.S.I.) - software de Classificação Hierárquica Implicativa e Coesiva - CHIC}

Aplicamos a Análise Estatística Implicativa (A.S.I.) através do uso do software de Classificação Hierárquica Implicativa e Coesiva - CHIC (Classification Hierarchique Implicative et Cohesitive - Versão 6.0,2012), que permite visualizar, organizar, construir modelos e explicar fenômenos associados aos dados, evidenciando o comportamento ou as imagens mentais de sujeitos em diferentes situações (Figura 3).

Cumpre destacar que as adaptações realizadas subvertem o processo da análise multivariada, já que partimos dos termos representativos gerados na etapa de mineração e das metas-categorias utilizando a A.S.I. para gerar os possíveis contextos de indagações sociais.

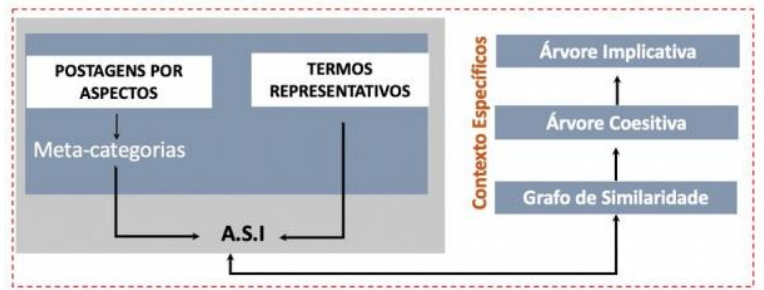

Figura 3 - Etapa de Contextos Narrados

Por exigência do software CHIC a estruturação dos dados é realizada de forma binária por meio de planilhas em MS Excel, com extensão csv, que separa os dados por vírgulas. Para a análise dos dados com o uso do CHIC selecionamos a opção "nós significativos", "cálculo longo". O tipo de implicação foi segundo a teoria clássica. O tipo de lei foi a lei binominal. Quanto mais próximo a 1,0 e acima de 0,7 melhor a confiabilidade dos dados. $O$ processamento dos dados possibilita a construção de árvores hierárquicas de similaridade e grafos implicativos (Figura 4). Vale lembrar que o índice de similaridade (Lerman and Manski, 1981) nos permite construir uma hierarquia ascendente cuja intensidade da implicação pode ser visualizada na árvore de interaç̧ão hierárquica.

Outro campo de visualização e análise da intensidade da implicação fornecido pelo CHIC é o Grafo implicativo (Figura 5), que permite ao pesquisador prefixar índices probabilísticos reconhecendo hierarquicamente seus fluxos de relações. 

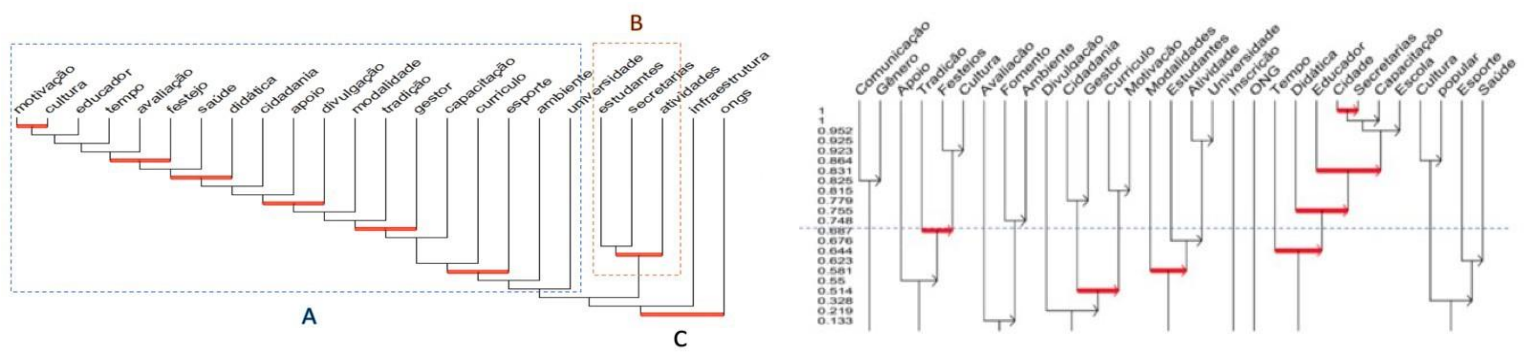

Figura 4 - Árvores de Similaridade e Árvores Coesitiva

É possível agrupar os dados a partir dos resultados de análise da similaridade entre as metacategorias, como também analisar a similaridade entre os termos representativos que foram organizados na composição dessa metacategoria. Outro recurso de visualização oferecido pelo grafo implicativo gerado pelo $\mathrm{CHIC}$, é a possibilidade do pesquisador selecionar diferentes camadas de relação entre os atributos de análise (de Queiroz et al., 2016). É possível agrupar os dados analisando a similaridade entre as meta-categorias, como também analisar a similaridade entre os termos representativos que foram organizados na composição dessa meta-categoria.

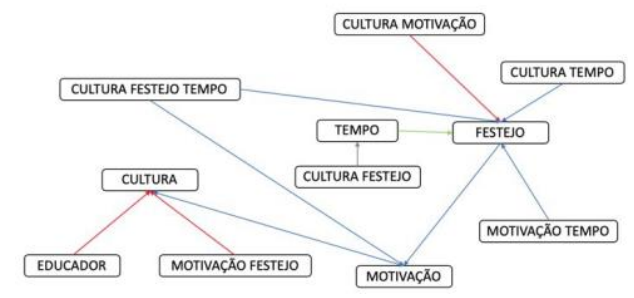

Figura 5 - Grafo Implicativo

\subsection{Interação com o Chatbot}

Para implementação dos instrumentos de interação do chatbot será utilizada a base de conhecimento gerada por essa proposta de inteligência social, ou seja, após identificarmos os contextos emergentes na fala da população sobre os problemas da cidade vamos instrumentalizar o Chatbot a discutir essas conjunturas com a população. Então, a partir do processo de escuta 0 chatbot realizará sua intervenção de duas formas: pela oferta de informações acerca da temática discutida ou por uma indagaçãa vincular. A proposta prevê a elaboração de um conjunto de indagações a partir de (3) três configurações básicas: explicação, exemplo e avaliação da pertinência das ponderações. Caso a conclusão do usuário indique a importância de sua manifestação, prevê-se o envio automático dessa formulação aos gestores públicos.

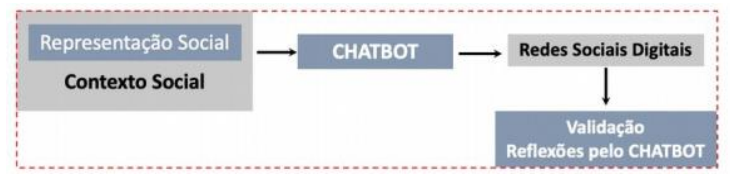

Figura 4 - Etapa de intervençãa pelo Chatbot

Um exemplo dessa funcionalidade pode ser proposto a partir da indagação oriunda do eixo educação para as meta-categorias Infraestrutura e Cidade:

Chatbot: Achas Rio Grande uma cidade educada? (explicação) 
Chatbot: Como é uma cidade educada? (exemplo)

Chatbot: Tua opinião é relevante para o problema? Sim/Não

Chatbot: Oi, tudo bem! Sabes das ações de educação da cidade? (explicação) Chatbot: Como agente pode promover educação pela cidade? (exemplo) Chatbot:

Tua opinião é relevante para o problema? Sim/Não

A finalização da conversa ocorre com a reflexão da validade da opinião que, ao ser confirmada, é enviada pelo chatbot como sugestão para os gestores públicos.

\section{Considerações Finais}

Todos os dias, os usuários do Twitter, Instagram e Facebook produzem um enorme volume de dados sobre o que estão fazendo, onde estão indo e como estão se sentindo. Não há como negar o comprometimento do cidadão que diante de uma situaçãa originária, age nas e com as redes digitais (re)concebendo sua relação com a cidade. A percepção manifesta atribui outros objetivos e motivos que aqueles previstos inicialmente por nestes artefatos, sendo eles tanto a cidade como as redes digitais (cidade-rede), por expandirem as relações do campo da conexão para a experiência de mobilização. É importante compreendermos que essas aventuras narradas, não constituem simplesmente retóricas de pedestres, elas verdadeiramente fundam territórios existenciais. E o modelo proposto por esse trabalho busca fornecer uma estratégia de análise desses ditos de forma que seus relatos representam organizadores de sujeitos e de lugares a partir do conjunto de vivências que descrevem.

\section{Agradecimentos}

Universidade Federal do Rio Grande, Instituto Federal do Rio Grande do Sul. O presente trabalho foi realizado, em parte, com apoio da Coordenação de Aperfeiçoamento de Pessoal de Nível Superior - Brasil (CAPES) - Código de Financiamento 001.

\section{Referências}

AFONSO, Ricardo A.; Tomas; OLIVEIRA, Alexandre A.; GARCIA, Vinicius C. Brscmm: Modelo brasileiro de maturidade para cidades inteligentes. Simpósio Brasileiro de Sistemas de Informação: 2013.

ALMEIDA JUNIOR, Oberdan Beck: um chatbot baseado na terapia cognitivo comportamental para apoiar adolescentes com depressão. Master's thesis, Universidade Federal de Pernambuco, 2017.

ALMEIDA, Maria Elizabeth Bianconcini T. M. O computador na escola: contextualizando a formação de professores-praticar e teoria, refletir a prática. 2000. https://doi.org/10.20873/uft.2447-4266.2019v5n1p202 , acesso em 15 maio 2019. 
ANASTASIADOU, Sofia; PAPADAKI, Zafeiria. Consumer's perceptions toward EService Quality, Perceived Value, Purchase and Loyalty Intentions. 2019. http://hdl.handle.net/11728/11391, Acesso em 15 maio 2019.

BERRONE, P.; RICART, J.E.; CARRASO, C.; RICART, R. IESE cities in motion index 2016. Retrieved 1 (2016): 2017.

COUTURIER, R.; A. BODIN; GRAS, R. A classificação hierárquica implicativa e coesiva. Manual CHIC: 2004. Disponível em: <http://dipmat.math.unipa.it/ grim/asi/asi_03_gras_bodin_cout.pdf>, Acesso15 maio 2019.

DE ALMEIDA COSTA, F.; DE SOUZA, C.A.P.; DE SIQUEIRA, C.A.F.; DARIO, R.V.; MARIA, Érica. Avaliação do ensino e da aprendizagem: uma análise de dados acerca dos discursos dos professores. Educação Matemática Pesquisa: 2018, no. 2.

FOTIADIS, Thomas A.; ANASTASIADOU, Sofia. Contemporary advanced statistical methods for the science of marketing: Implicative Statistical Analysis vs Principal Components Analysis. 2019.

GABRYS, Jennifer. Programming environments: environmentality and citizen sensing in the smart city. Environment and Planning: Society and Space: 2014. Pp.30-48

GASPAR, J. V.; I. S. C. AZEVEDO; C. S. TEIXEIRA. Analise do Ranking Connected Smart Cities. Congresso Internacional de Conhecimento e Inovação. 2016.

GIORDANO, Cássio. Concepções sobre estatística: um estudo com alunos e professores do ensino médio. 2019. http://hdl.handle.net/10481/55062 Acesso em 15 maio 2019.

GOODFELLOW, Ian; BENGIO, Yoshua; COURVILLE, Aaron. Deep learning. MIT press: 2016.

GRAF, Hans Daniel, et al. Four different ways to build a chatbot about movies. In SwissText 2017: 2nd Swiss Text Analytics Conference, Winterthur, 9. Juni 2017, 2017.

GRANIER, Benoit; KUDO, Hiroko. How are citizens involved in smart cities? Analysing citizen participation in Japanese Smart Communities. Information Polity: 2016. Pp. 61-76.

HAN, J.; K., MICHELINE; PEI, J. Data mining concepts and techniques third edition. Morgan Kaufmann: 2011.

HE, K., Zhang, X., REN, S.,SUN, J. (2016). Deep residual learning for image recognition. In Proceedings of the IEEE conference on computer vision and pattern recognition (pp. 770-778). 
HOSSAIN, M. D., SOHEL, F., SHIRATUDDIN, M. F., \& LAGA, H. (2019). A comprehensive survey of deep learning for image captioning. ACM Computing Surveys (CSUR), 51(6), 118.

KOMNINOS, Nicos. The age of intelligent cities: Smart environments and innovationfor-all strategies. Routledge, 2014.

KUYVEN, Neiva L.; ANTUNES, Carlos A.; VANZIN, Vinicius J. de B.; SILVA, João L. T. da; KRASSMANN, Aliane L.; TAROUCO, Liane M. Rockenbach. Chatbots na educação: uma Revisão Sistemática da Literatura. RENOTE 16, no. 1, 2018.

LERMAN, Steven; MANSKI, Charles. On the use of simulated frequencies to approximate choice probabilities. Structural analysis of discrete data with econometric applications: 1981. Pp.305-319.

LIBÂNEO, José Carlos. $O$ ensino da Didática, das metodologias específicas e dos conteúdos específicos do ensino fundamental nos currículos dos cursos de Pedagogia. Revista Brasileira de Estudos Pedagógicos: 2010. Pp.229

PAEZ CANDELARIA, Yordanys; DEL CAMPO, Nelsa M. S.; MATAMOROS, Larisa Z.. Statistical analysis involving the determination of prognosis factors for the nutritional state of the severely ill patient at discharge. MediSan: 2018, pp. 431-440.

POPLIN, R., Varadarajan, A. V., BLUMER, K., Liu, Y., MCCONNELL, M. V., Corrado, G. S., \& Webster, D. R. (2018). Prediction of cardiovascular risk factors from retinal fundus photographs via deep learning. Nature Biomedical Engineering, 2(3), 158.

RAKOTOMALALA, Hery Frédéric; RALAHADY, Bruno Bakys; Totohasina, André. A Novel Cohesitive Implicative Classification Based on $\mathbf{\$} \mathbf{M} \mathbf{M}_{-}\{\mathbf{G K}\} \mathbf{\$}$ and Application on Diagnostic on Informatics Literacy of Students of Higher Education in Madagascar. Third International Congress on Information and Communication Technology. Springer, Singapore: 2019.

RODRIGUES, Marcelo Rivelino. Estudo sobre as concepções de professores do ensino básico em relação a aleatoriedade e probabilidade. 2018.

SÔNEGO, A. A.; BERNARDINI, A.A; POZZEBON, E. Chatbots: Uma Análise Bibliométrica do Estado da Arte da Literatura. ARTEFACTUM-Revista de estudos em Linguagens e Tecnologia: 2018.

SYSTEMS, U. (2018). Urban systems. [Online; acessado abril, 2019].

VENDRÚSCOLO, Juliana de B. Garcia; MORÉ, Rafael P. Ocampo. Contribuições Da Inteligência Artificial nos Sistemas de Informação de Apoio à Gestão Universitária. 2018. 